\title{
Yet again, quantum indeterminacy is not worldly indecision
}

\author{
Alberto Corti ${ }^{1,2}$ (D) \\ Received: 21 February 2020 / Accepted: 16 January 2021 / Published online: 6 March 2021 \\ (c) The Author(s) 2021
}

\begin{abstract}
It has been argued that non-relativistic quantum mechanics is the best hunting ground for genuine examples of metaphysical indeterminacy. Approaches to metaphysical indeterminacy can be divided into two families: meta-level and object-level accounts. It has been shown (Darby in Australasian Journal of Philosophy 88(2):27-245, 2010. https://doi.org/10.1080/00048400903097786; Skow in Philosophical Quarterly $60(241): 851-858,2010)$ that the most popular version of the meta-level accounts, namely the metaphysical supervaluationism proposed by Barnes and Williams (Oxford Studies in Metaphysics, Oxford University Press, Oxford, pp 103-148, 2011), fails to deal with quantum indeterminacy. Such a fact has been taken by many as a challenge to adapt supervaluationism to quantum cases. In this paper, I will focus on the very last of these attempts, i.e. the situation semantics account proposed by Darby and Pickup (Synthese 1-26, 2019). After having shown where quantum indeterminacy arises and having surveyed the assumptions endorsed by the participants of the debate, I turn to Darby and Pickup's proposal. I argue that, despite the machinery introduced, their account still fails to account for quantum indeterminacy. After considering some possible counterarguments, I suggest in the conclusion that one can plausibly extend the argument to those meta-level approaches that treat quantum indeterminacy as worldly indecision.
\end{abstract}

Keywords Metaphysical indeterminacy · Meta-level accounts · Quantum mechanics $\cdot$ Metaphysics of science $\cdot$ Situation semantics

\section{Introduction}

Metaphysical indeterminacy (henceforth 'MI') is the idea that indeterminacy is a feature of the world itself, rather than a feature imputable to the words we use (Fine 1975)

$凶$ Alberto Corti

a.corti1@campus.uniurb.it

1 Department of Philosophy, University of Geneva, Rue de Candolle 5, 12111211 Geneva 4, Switzerland

2 Department of Pure and Applied Sciences, University of Urbino, Via Timoteo Viti 10, 61029 Urbino, Italy 
or to our knowledge (Williamson 1994). MI has often been regarded as a priori incoherent. Such an attitude has been encouraged by three main facts. First, many thought that MI necessarily allows the possibility of indeterminate identities. Given the strength of Evan's (1978) argument against such a position, the majority of philosophers have concluded that MI is impossible. Second, many's attitudes on MI were influenced by authoritative philosophers like Russell (1923) and Lewis (1986), who explicitly banned MI as conceptually contradictory or, if coherent, blatantly impossible. Third, there were no positive reasons for thinking that MI was conceivable in the first place. Indeed, precise and convincing accounts of MI were nowhere to be found, leading many to the conclusion that MI is inconceivable.

The attitude toward the possibility of MI now seems to be more relaxed than before, especially because logically clear accounts of MI have been put forward in the literature. Moreover, many authors have argued that these accounts help make sense of different phenomena. ${ }^{1}$

As a matter of fact, many think that the most compelling reason for taking MI seriously is that some interpretations of non-relativistic quantum mechanics, plus some extra assumptions, seem to suggest a genuine case of it. Even if the first examples of quantum MI proposed ${ }^{2}$ are no longer seen as genuine, that the properties of quantum objects are metaphysically indeterminate is still a live option (Bokulich 2014; Wolff 2015; Calosi and Wilson 2018).

Following Wilson (2013), one may divide the accounts of MI available in the literature into two broad categories: ${ }^{3}$

- Meta-level accounts: usually, these approaches consider MI as worldly 'indecision' between different possible, mutually incompatible, precise states of affairs. In other terms, $p$ is metaphysically indeterminate iff (i) there are at least two possible descriptions of a state of affairs involving $p$, i.e. $p$ and $\neg p$, and (ii) these possible descriptions that do not contain in themselves any semantic nor epistemic indeterminacy fail in both determinately representing and misrepresenting the actual world. The details vary slightly across models, but the general idea remains the same: a state of affairs is metaphysically indeterminate when the world itself is unsettled between (at least) two possible determinate states. Examples of metalevel accounts can be found in Akiba (2004), Barnes and Williams (2011) and Darby et al. (2017).

- Object-level accounts: this family of accounts claims that there are three possible states of affairs: the state that $p$, that $\neg \mathrm{p}$ and the state that it is indeterminate whether p. MI is thus considered as a third state of affairs that cannot be reduced nor be

\footnotetext{
1 Future (Barnes and Cameron 2009) and fictional (Darby et al. 2017) states of affairs are examples among others.

2 Notably the one proposed by Lowe (1994) as a counterexample of Evans' argument. The contemporary general agreement is that quantum mechanics does not conclusively support indeterminate identity. For a survey on identity in quantum mechanics see French and Krause (2006).

3 What follows is a rough and ready description of the main intuition that most of these approaches to MI try to capture rather than an exhaustive presentation of every view. An important exception is the view presented in Torza (2017). Such a view is a meta-level account, even though it does not involve any worldly indecision; rather, the indeterminacy is considered as due to the incompleteness of the ersatz world that represents reality.
} 
understood in terms of the two definite states $p$ and $\neg$ p. Arguably, Tye (1990) is one of the progenitors of this approach to MI, albeit his account seems not so popular anymore for different reasons. ${ }^{4}$ Other object-level accounts of MI can be found in Smith and Rosen (2004) and Wilson (2013).

The contemporary research in MI seems to be oriented toward the former family of accounts rather than the latter. ${ }^{5}$ In what follows, I focus on meta-level treatments of indeterminacy and how they deal with examples taken from non-relativistic quantum mechanics. In particular, I focus on the account recently developed in Darby et al. (2017) and Darby and Pickup (2019). I provide background on where and under what assumptions quantum indeterminacy is supposed to arise in Sect. 2, while Sect. 3 is devoted to a presentation of Darby and Pickup's account. ${ }^{6}$ Then, I argue in Sect. 4 that this way of modelling metaphysical indeterminacy fails to account for quantum phenomena, even granting all the assumptions requested by Darby and Pickup's view. After considering possible replies to the argument here presented (Sect. 5), I draw what I take to be the moral of all of this, namely, that quantum superposition cannot be understood as worldly indecision between definite states of affairs (Sect. 6).

\section{Where quantum indeterminacy lies}

That quantum mechanics offers a genuine example of MI is debatable. Indeed, such a claim relies on five main assumptions which are, by themselves, highly controversial. In this section, I briefly discuss them in turns.

First, the relevant literature concerning quantum MI assumes as a background an 'orthodox' interpretation of quantum mechanics. ${ }^{7}$ It must be acknowledged that some of the main players of the debate have argued that MI also arises in other interpretations $^{8}$ as well; still, no complete and satisfying investigation in this sense has been put forward yet. Since orthodox quantum mechanics is seen as an instrumental view, the second assumption held is that being realist or antirealist is a stance one assumes toward a theory, rather than something embedded in the theory itself (following McMullin 1984).

\footnotetext{
4 e.g. The open rejection of classical logic and the (alleged) difficulty of overcoming Evans' argument against indeterminate identity.

5 Such a fact may be surprising if one agrees with Wilson (2013) that meta-level accounts somehow rely on the very same intuition that guided the critics of MI, i.e. that MI is not a particular state of affairs, and that it does not make sense if not understood as unsettledness between determinate states of affairs.

6 Readers already familiar with quantum indeterminacy and Darby and Pickup's account may safely skip these two sections.

7 It is not completely clear what they mean by 'orthodox' interpretation. A more accurate description would be that they try out 'interpretation-neutral discussions of the ontology of QM' (Wallace 2019, p. 3). A charitable way of interpreting their claim is that of reading 'orthodox' interpretation as 'textbook quantum mechanics', i.e. the currently taught quantum mechanics based on the Dirac-von Neumann formulation of the theory.

8 In particular in GRW (Calosi and Wilson 2018), Relational QM (Calosi and Mariani 2020) and Many Worlds interpretation (Wilson 2020).
} 
The third assumption is that Hermitian operators correspond to properties instantiated by quantum systems $;{ }^{9}$ in particular, the relation between the properties instantiated and their mathematical description is captured by the 'Eigenstate-Eigenvalue link' (EEL): ${ }^{10}$

(EEL) A quantum system has a definite value $v$ for an observable $O$ iff it is in an eigenstate of $\hat{O}$ having eigenvalue $v$.

This principle bridges the mathematical and the metaphysical descriptions. Under the assumption that the properties of a system (i.e. its observables) are modelled in quantum formalism by Hermitian operators, (EEL) gives us the sufficient and necessary conditions under which we can claim that a quantum system instantiates some properties: if a property $O$ is represented by a Hermitian operator $\hat{O}$, the system instantiates that property if and only if its state is an eigenstate of $\hat{O}$.

There are many cases in which a system fails to be in an eigenstate of some operator. Let us consider spin- $\frac{1}{2}$ along different directions. Given some properties of the mathematical vector space in which spin properties are represented, if a system is in a definite state of, say, spin up along the $x$-axis $\left(|\uparrow\rangle_{x}\right)$, it is necessarily in a superposed state of spin along the $z$-axis:

$$
|\psi\rangle=|\uparrow\rangle_{x}=\frac{1}{\sqrt{2}}\left(|\uparrow\rangle_{z}+|\downarrow\rangle_{z}\right)
$$

Since $\frac{1}{\sqrt{2}}\left(|\uparrow\rangle_{z}+|\downarrow\rangle_{z}\right)$ is not an eigenvector of the operator $\sigma_{z}$ (i.e. the operator correspondent to the property 'having spin along the $z$-axis'), when the system is in $|\psi\rangle$, it does not have a definite value of spin along the $z$-axis. Friends of quantum indeterminacy claim that when a system is in a state like $|\psi\rangle$, or more generally, in a state that is not an eigenvector of some Hermitian operator, the system indeterminately instantiates the property that corresponds to that operator. These kind of states are (allegedly) responsible for the arise of quantum indeterminacy: when a quantum system is in one of these states, the instantiation of one of its properties might be indeterminate.

The fourth assumption is that such indeterminacy is metaphysical rather than semantic or epistemic. It is not semantic because equations are sharp and precise, and none of the imprecision connected with the ambiguities of natural languages is involved. ${ }^{11}$ It is not epistemic because some mathematical theorems ${ }^{12}$ show formally that if you try

\footnotetext{
9 This assumption has been contested, for example, in Daumer et al. (1996); as a matter of fact, this assumption excludes MI from some interpretations that explicitly reject it.

10 Or some of its variants, as it is discussed in (Lewis 2016, Ch. 4).

11 Some might be tempted to point out that some authors, most notably Reichenbach, suggested that quantum mechanics is semantically indeterminate, in some sense or another. Although I would agree with that, my reply is that such indeterminacy is not only semantic, i.e. a specification of the terms used do not wash away, so to speak, the indeterminacy. That (alleged) semantic indeterminacy in quantum mechanics reflects a more fundamental kind of indeterminacy, one might be tempted to call it 'metaphysical', is acknowledged for instance by Reichenbach (1975, p. 94) himself : "The deficiencies [i.e. that some propositions have an indeterminate truth-value] must rather be regarded as the linguistic expression of the structure of the atomic world, which thus is recognized as intrinsically different from the macro-world, and likewise from the atomic world which classical physics had imagined."

12 The most known formal results are those of: Gleason (1957), Kochen and Specker (1967) and Pusey et al. (2012).
} 
to cheat, so to speak, by assigning a definite value to all the properties, you contradict the empirically tested predictions of quantum mechanics. The general conclusion is then that such indeterminacy must be metaphysical.

Note finally that (EEL) per se is not enough for claiming that the system indeterminately instantiates some properties. As Glick (2017) argues, one may take the (EEL) as saying that when the state of a system is not an eigenvector of an operator, it lacks the corresponding property altogether. Then, the fifth assumption taken by the participants of the debate is what I dub 'Property Nonetheless' (PN):

(PN) A quantum system always instantiates its (possible) observables (i.e. those properties that can be measured and represented mathematically through a Hermitian operator). ${ }^{13}$

This principle should guarantee that a system always instantiates its properties, making room for the possibility of indeterminacy. Indeed, (PN) and (EEL) jointly implies that when the system is not in an eigenstate of one of its observables, it does not possess a definite value for the corresponding properties. The fact that these values are not definite allows us to interpret it as if the system indeterminately instantiates the property mentioned above.

Most of the assumptions above are controversial. The (EEL) has been strongly criticized in different interpretations of quantum mechanics (Wallace 2019; Albert and Loewer 1996); the claim that indeterminacy is not semantic nor epistemic might be resisted (either assuming an empiricist stance toward science or accepting some $\psi$-epistemic interpretation of quantum mechanics). Insofar as, in general, issues on quantum mechanics tend to be highly controversial, such a list could continue forever. Since these assumptions are necessary for arguing that quantum mechanics leaves open the possibility of MI, reasonably, they are also endorsed by Darby and Pickup. So far as the paper aims at showing that Darby and Pickup's account fails to account for quantum indeterminacy I uncritically endorse, for the sake of argument, the assumptions above in what follows.

Having considered the assumptions needed in order to claim that quantum mechanics offers a genuine example of MI, we now turn to how meta-level approaches deal with quantum MI.

\section{Meta-level accounts of quantum indeterminacy}

Barnes and Williams (2011) proposed a supervaluationist account of MI. According to them, when there is MI, there are at least two ersatz possible worlds (that do not contain any semantic or epistemic indeterminacy in themselves) such that they both fail to represent reality correctly. However, these possible worlds do not determinately misrepresent reality either. So, if it is metaphysically indeterminate if $p$, then there is a possible world in which $p$ obtains and a possible world in which $\neg p$ obtains,

\footnotetext{
13 Note that the principle does not require that the properties of quantum systems are definite at all the times.
} 
but it is in principle ${ }^{14}$ impossible to say which of these represent the concrete actual world. It has been argued that their proposal fails to account for the indeterminacy that arises in quantum mechanics: as Darby (2010) and Skow (2010) independently showed, the Kochen-Specker theorem implies that there is at least one case in which it is impossible to assign simultaneously (in a context-independent way) a definite value to all the properties of a quantum system. In Barnes and Williams' account, the ersatz possible worlds are entirely defined and determinate; that is, in every possible world, all the properties of every system always have a definite value. Since the Kochen-Specker theorem shows that there is at least one quantum system such that it is impossible to assign definite values to all its properties, no possible worlds (of the kind employed in Barnes and Williams' proposal) may be used to account for the indeterminacy of this quantum system. Since there is at least a quantum system whose indeterminacy cannot be accounted for using Barnes and Williams' view, their account of MI is not apt for explaining quantum indeterminacy in general.

The failure of Barnes and Williams' account has been taken by many as a challenge to develop a supervaluationist treatment of MI able to deal with quantum cases. ${ }^{15}$ In what follows, I focus only on the most recent attempt to develop a supervaluationist account of MI, namely the view proposed by Darby and Pickup (2017, 2019).

The account proposed by Darby and Pickup consists of using a situation semantics instead of the machinery of possible worlds. The core idea of situations semantic is that the truth-value of a proposition ${ }^{16}$ has to be assessed relative to a situation; where situations are parts of our concrete actual world, i.e. situations are non-representational concrete truth-makers. In the situation semantics framework, propositions are not true or false simpliciter; rather, their truth-value is relative to the situation at which they are evaluated. ${ }^{17}$ Hence, truth (and falsehood) simpliciter is replaced with 'truth (falsehood) at a situation.'

Insofar as situations are intrinsically partial, they do not tell everything about a certain state of affairs. In those situations that lack an aspect of reality, the proposition expressing that part is taken by Darby and Pickup as lacking a truth-value. ${ }^{18}$ An example will illustrate how situation semantics works. Take a statement like 'Alice is playing with

\footnotetext{
14 That is, it is not a matter of knowledge. One may doubt, following the argument proposed by Akiba (2015), that the account presented is not metaphysical, but semantic or epistemic at best. Even if the same argument can be applied to the Darby and Pickup account, I will not belabour this point in what follows.

15 See for example: Torza (2017); Mariani, Michels, and Torrengo, (manuscript presented at the 4th Annual Conference of the Society for the Metaphysics of Science).

16 Darby and Pickup explicitly use 'propositions' instead of the more neutral 'sentences.' For the sake of argument, I also use 'propositions' in what follows.

17 Darby and Pickup describe situations as parts of possible worlds. Since their account is explicitly a modification of the one proposed by Barnes and Williams, one may think that situations are ersatz, as Barnes and Williams' possible worlds are. In previous drafts of the paper, I assumed such an interpretation of Darby and Pickup's account. As an anonymous referee makes me notice, though, it is more charitable to assume that Darby and Pickup's situations are parts of the concrete world.

18 Insofar as they do not offer a precise logic of their situation semantics, it is not entirely clear how to model it. One possibility is that of building a semantics with a partial evaluation function; another is that of introducing a third truth value. A final possibility is that of following Levesque's (1984) account, according to which it is not possible to assign a truth value (in certain situations) to some propositions. The last possibility seems to be closest to what Darby and Pickup's have in mind. Alas, such a semantics is complicated and baroque already at first-order. Even if there are reasons to think that the logic needed by
} 
her dog'. Such a proposition is true if Alice is playing with her dog in the relevant situation and it is false if she is jogging (or doing something different than playing with her dog) in the relevant situation. In those situations in which there is no Alice, like in the situation in which there is a ski race on a mountain distant from Alice, the proposition 'Alice is playing with her dog' lacks a truth-value. Following Barnes and Williams, Darby and Pickup try to model indeterminacy as a matter of truth-values in situations which are candidates for representing actuality. The criteria of 'candidates for representing actuality' employed seems to be the following: a situation is a candidate for representing actuality if it does not determinately misrepresent ${ }^{19}$ the actual world. In the previous example, if Alice is playing with her dog in the actual world, then any situation in which the proposition 'Alice is playing with her dog' is true is a candidate for representing actuality; all the situations that determinately misrepresent the actual world, i.e. those in which Alice is doing something else, are not candidates for representing actuality. Given the fact that they accept that propositions can lack a truth-value at a situation, also situations in which there is no Alice will be candidates for representing actuality; obviously, this is true only if these situations do not misrepresent the actual world in other regards. For example, if in the actual world, while Alice is playing with her dog, there is a ski race on some distant mountain, then both the situation with Alice playing and the situation in which there is a ski race going on are candidates for reality.

Using the machinery of situation semantics, they propose to model MI as a genuine disagreement between those situations that are candidates for representing actuality. When a state of affairs is determinate, the relevant situations, i.e. those that are candidates for representing actuality that describe such a state of affairs, never disagree. In the previous example, for instance, the situation with Alice playing with her dog in some places does not contradict the situation with a ski race going on in some distant mountain. However, they claim that, if there is MI, then we have different situations that disagree on what reality is like, despite the fact that all of them are candidates for representing actuality. ${ }^{20}$ To capture such a disagreement, they propose the following criteria:

Determinacy A proposition $p$ is determinate iff it is true in some situation which is a candidate for representing reality and false in no such situation.

Footnote 18 continued

Darby and Pickup account is much more complex, if feasible at all, than what the authors explicitly show, I will not press this point further.

19 They do not explain what 'determinately misrepresenting' means despite the prima facie ambiguity of the term. I take its meaning to be the following: given a proposition $p$ that is determinately true in the actual world, all the situations that make true a proposition that, if conjoined with $p$, form a logical contradiction, determinately misrepresent the actual world. For example, suppose that in the actual world Alice is playing with her dog, so that 'Alice is playing with her dog' is true. All the situations that verify a proposition that, together with 'Alice is playing with her dog', entails a contradiction, determinately misrepresent the reality. Note that since situations are concrete, it is inappropriate to say that they 'misrepresent' the actual world, because they are supposed to be parts of it. Nonetheless, I adopt in what follows Darby and Pickup's explicit terminology.

20 Note cursorily that, although the situations that are candidates for representing actuality may disagree with each other, they lack any contradiction in themselves. Indeed, no situation verifies $p$ and $\neg p$ at the same time. 
Indeterminacy A proposition $p$ is indeterminate iff it is true in some situation which is a candidate for representing reality and false in some other such situation. (Darby and Pickup 2019, p. 9)

According to Darby and Pickup's account, a proposition is determinately true when it is not false at any situation that is a candidate for representing actuality and true in at least one. ${ }^{21}$ In contrast, a proposition is indeterminate when there are at least two situations that are candidates for representing actuality, and the proposition is true at one of them and false at the other.

Again, an example helps us to understand better how the machinery works. Let us suppose that it is metaphysically indeterminate whether Alice is playing with her dog. In the set of situations which do not determinately misrepresent the actual world, there will be many situations that do not say anything about Alice; in respect of these situations, the proposition 'Alice is playing with her dog' simply lacks any truth-value. All the other situations will be of two kinds. Some situations which are candidates for representing reality make true that Alice is playing with her dog; however, the same proposition will be false at other situations which are candidates for representing actuality. Given that the situations in which the proposition is true are candidates for representing actuality as good as those in which the proposition is false, it is metaphysically indeterminate whether Alice is playing with her dog in the actual world. $^{22}$

Let us turn now to how they deal with quantum superposition. They consider the wellknown example of Schrödinger's cat: a cat is closed in a diabolic box that contains a device that releases a poisonous gas if an atom of isotope uranium decays. If the atom decays, the cat drops dead; if it does not decay, the cat survives. According to quantum mechanics, the system evolves in a way that, after a certain time, the atom will be in a superposition of being decayed and being not; since the atom and the cat compose a bigger system, the upshot of the thought experiment is that also the cat ends up in a superposition of being alive and dead. How can we make sense of such indeterminacy? This is Darby and Pickup's proposal, which I quote at length:

Our model can deal with this case in a natural way. Consider the following three situations, each of which is a candidate for representing actuality: $\mathrm{s}_{1}$, a situation in which the cat is alive, $\mathrm{s}_{2}$, a situation in which the cat is dead, and $\mathrm{s}_{3}$, the fusion of $s_{1}$ and $s_{2}$. In $s_{1}$, it is true that the cat is alive and false that it is dead. In $s_{2}$ it is true that the cat is dead and false that it is alive. So, what status do these propositions have? According to our definition, they are indeterminate, because there is a situation which is a candidate for representing actuality in which they are true and another situation which is a candidate for representing actuality in which they are false. What is the case in $s_{3}$ ? It seems $s_{3}$ is overdetermined with respect to the cat's mortality, as parts of $s_{3}$ disagree about whether the

\footnotetext{
${ }^{21}$ And conversely it is determinately false when it is not true at any situation that is a candidate for representing actuality and false in at least one.

22 Darby and Pickup's account is much richer and articulated than my brief summary. Indeed, they do present some details that, since they are not crucial here, I have skipped. Moreover, they present and discuss some alternatives to their account. Since they do acknowledge explicitly why the variants of their situations account fails, I will stick to the main account only.
} 
cat is alive. Specifically, $s_{2}$ precludes the proposition that the cat is alive and $\mathrm{s}_{1}$ precludes the proposition that the cat is dead. So, following our argument above, the proposition that the cat is alive is neither true nor false in $s_{3}$ (as is the proposition that the cat is dead). The indeterminacy involved in superposition is therefore dealt with. $(2019$, p. 16)

One of the crucial aspects of their account that emerges in the quotation is the following: if two contradictory propositions (e.g. $p$ and $\neg p$ ) are going to be verified in the same situation, then they both lack a truth-value at that situation. Such a detail is crucial for avoiding the Kochen-Specker result. Recall why Barnes and Williams' account fails with quantum indeterminacy: since they use complete possible worlds that do not contain any indeterminacy in themselves, they must attribute definite values to all of a quantum system's properties at the same time. Darby and Pickup's account instead, successfully manages to avoid such a contradiction. Indeed, they argue that different situations describe different properties of the system but, crucially, no incompatible properties will receive a definite value in the same situation. Insofar as a situation might be silent on some aspect of reality, there is no need to assign definite values to all the properties of a quantum system. Using disagreeing situations, and avoiding any contradiction arising from assigning values to incompatible properties in the same situations, their machinery of situations accounts for quantum indeterminacy.

\section{A situation in which situation semantics fails}

Albeit prima facie Darby and Pickup's account seems to accommodate cases of quantum indeterminacy, I argue now that it is not the case.

Let us start by assuming that in the actual world an electron is in the state:

$$
|\psi\rangle=\frac{1}{\sqrt{2}}\left(|\uparrow\rangle_{z}+|\downarrow\rangle_{z}\right)
$$

Note that according to the formalism of quantum mechanics, state (2) is mathematically equivalent to:

$$
|\psi\rangle=|\uparrow\rangle_{x}
$$

Indeed, being in a superposition on the $z$-axis means having a definite value for the $x$ component of spin. ${ }^{23}$ Note that this implies that being in spin up along the $x$-axis means being in a superposition of the $z$-axis; in other terms, $|\uparrow\rangle_{x}$ and $\frac{1}{\sqrt{2}}\left(|\uparrow\rangle_{z}+|\downarrow\rangle_{z}\right.$ are mathematically equivalent ways of describing the same vector (i.e. the vector is represented in different bases of the Hilbert space).

\footnotetext{
23 To be more specific, it depends on how one labels the vectors in the Hilbert space used for representing spin states. What is true is that being in a superposition of an axis means being in a state that is an eigenvector of some operator that represents the property of having spin along another axis. We assume that the vectors that are equal to a superposition of the $z$ component of spin with expansion coefficients $\frac{1}{\sqrt{2}}$ are spin up and spin down along the $x$-axis.
} 
Darby and Pickup's account deals with superposition in the following way: when a system is superposed on an axis, there is a situation in which the system has spin up, and a situation at which it has spin down. Therefore, if in the real world our electron is in state $|\psi\rangle$, then there are at least two situations that are candidates for representing actuality: ${ }^{24}$ at $s_{1}$ the particle has spin-up along the $z$-axis and at $s_{2}$ it has spin-down along the $z$-axis. Darby and Pickup propose to capture the two properties that the electron could have through a couple of propositions. The proposition expressing the fact that the electron has spin up is true at the first situation and false at the second; the opposite is true for the proposition expressing the property of having spin down along the $z$-axis. Albeit they do not specify what such a proposition is, I propose to consider the following:

$\left(p_{z^{+}}\right)$The system is in a state that is an eigenvector of the operator $\sigma_{z}$ with a corresponding eigenvalue $+1 .^{25}$

This proposition, which captures the fact that the system has spin up along the $z$-axis, is true at $s_{1}$, and false at $s_{2}$ since it makes true a different proposition that entails the falsehood of $\left(p_{z^{+}}\right) .{ }^{26}$ Note that $\left(p_{z^{+}}\right)$is also false when the system is in a superposed state like (1). ${ }^{27}$ Indeed, a superposed state of eigenvectors is not itself an eigenvector of its corresponding operator. That is to say that a superposition of the eigenvectors (with different eigenvalues) of the operator $\sigma_{z}$, is not itself an eigenvector of $\sigma_{z}$ (but crucially, it will be an eigenvector of some other operator; in the case at hand, of $\sigma_{x}$ when the expansion coefficients of the superposition are $\frac{1}{\sqrt{2}}$ ).

Let us focus now on their criteria of 'candidate for representing actuality'. A situation is a candidate for representing actuality if it does not determinately misrepresent the actual world. An example is the situation $\mathrm{s}_{3}$ that they explicitly accept, i.e. the situation that should verify both $s_{1}$ and $s_{2}$. In this situation, $\left(p_{z^{+}}\right)$and $\left(p_{z^{-}}\right)$both lack a truth value. However, given the definition of 'candidate for representing actuality,' we can find many other situations concerning our electron that Darby and Pickup do not consider. Take the proposition:

$\left(p_{x^{+}}\right)$The system is in a state that is an eigenvector of the operator $\sigma_{x}$ with a corresponding eigenvalue +1 ,

There will be a situation $\left(s_{4}\right)$ candidate for actuality which makes true only $\left(p_{x^{+}}\right)$.

At this stage of the argument, I am going to endorse the following principle, which I dub the principle of 'equivalent candidates for representing actuality' (ECA henceforth):

\footnotetext{
${ }^{24}$ Note that there is also a third situation that is a fusion of $s_{1}$ and $s_{2}$, at which the truth value of the propositions that capture the particle having spin up and spin down along the $z$-axis both lack a truth value.

25 I considered, as is common in the literature on quantum mechanics, having spin up (down) and having eigenvalue $(-)+1$ the same property.

26 The situation s2 makes true only the proposition $\left(p_{z^{-}}\right)$: 'The system is in a state that is an eigenvector of the operator $\sigma_{z}$ with a corresponding eigenvalue $-1^{\prime}$. Given some semantic assumptions held by Darby and Pickup and some rules of linear algebra, the truth of $\left(p_{z^{-}}\right)$entails the falsehood of $\left(p_{z^{+}}\right)$, and vice versa.

27 On the contrary, in Darby and Pickup's account a proposition like 'The system has spin up along the $z$-axis' would lack a truth-value in the situations in which the system is superposed. As it will be clear later on, distinguishing propositions that express a mathematical description of reality from those expressing a metaphysical one is crucial to run the argument against Darby and Pickup's view.
} 
(ECA) Consider a situation $s_{1}$ that is a candidate for representing actuality and verifies only a proposition $p_{1}$ which contains a mathematical object $o_{1}$. Any other situation $\mathrm{s}_{2}$ that differs from $\mathrm{s}_{1}$ only in that it makes true a proposition $p_{2}$ which is obtained by simply replacing $o_{1}$ with $o_{2}$, where the latter is a mathematical object equivalent to the former (i.e. $o_{1}=o_{2}$ ), is also a candidate for representing actuality.

Such a principle seems to be intuitively reasonable. Let us see how it works by presenting a toy example. Suppose it is metaphysically indeterminate how many oranges there are in the fridge; assume further that there might just be either three or four. According to Darby and Pickup's view, there is a possible situation in which there are three oranges, and a possible situation in which there are four, but neither describes correctly nor misrepresents the actual world (and therefore the propositions 'there are three oranges in the fridge' and 'there are four oranges in the fridge' are indeterminate, being true and false in at least one situation). The principle (ECA) simply guarantees that if the possible situation in which there are three oranges is a candidate for representing actuality, then also the situations that verify respectively only the propositions 'there are two plus one oranges in the fridge' or 'there are four minus one oranges in the fridge', and so on, are candidates for representing actuality. All of this might seem superfluous, but it is not. In everyday examples like the one above, there will usually be a unique situation that makes true every mathematically equivalent proposition. However, it is better to assume that it is not the case for quantum phenomena. Darby and Pickup (2019, p. 11) explicitly say that a situation that verifies a proposition concerning, say, spin along the $x$-axis may not verify any proposition concerning spin along a different direction. To avoid begging the question against their view, I assume that there are different situations that make true (or false) propositions concerning spin in different directions. ${ }^{28}$

Coming back to quantum indeterminacy, we have seen that the state spin up along the $x$-axis is mathematically equivalent to being in a superposition of spin up and down along the $z$-axis. That is, they are the very same state from a mathematical point of view. ${ }^{29}$ The same can be said of the latter states, i.e. being in spin up and down along the $z$-axis means being in a superposition of spin up and down along the $x$-axis. To put it explicitly:

$$
\begin{aligned}
& |\uparrow\rangle_{z}=\frac{1}{\sqrt{2}}\left(|\uparrow\rangle_{x}+|\downarrow\rangle_{x}\right) \\
& |\downarrow\rangle_{z}=\frac{1}{\sqrt{2}}\left(|\uparrow\rangle_{x}-|\downarrow\rangle_{x}\right)
\end{aligned}
$$

\footnotetext{
28 Mainly because Darby and Pickup (2019, p. 11) explicitly acknowledge that this feature of situation semantic is crucial to avoid arguments based on the Kochen-Specker theorem. Moreover, note that the argument that follows could be restated, in a slightly different form, even granting that there is a unique situation that verifies every proposition concerning the mathematically equivalent representation of spin. I thank an anonymous referee for pressing me on this point.

29 This is due to the properties of the complex vector space in which states of spin live in. What said so far can be showed formally, even though there is no need to do it here.
} 
Give (ECA), if a situation that is a candidate for representing actuality makes true a proposition that captures the fact that the system is in state spin up (down) along the $z$-axis, then also the situation that verifies a proposition describing the system in a superposed state of spin up plus (minus) spin down the $x$-axis must be a candidate for representing actuality.

To sum up, when a system in the actual world is in state spin up along the $x$-axis, and thus in a superposition of spin along the $z$-axis, there are three situations that are candidates for representing actuality that Darby and Pickup explicitly accept:

$$
\begin{aligned}
& \mathrm{s}_{1}:\left\{p_{z^{+}}\right\} \\
& \mathrm{s}_{2}:\left\{p_{z^{-}}\right\} \\
& \mathrm{s}_{3}:\left\{p_{z^{+}}, p_{z^{-}}\right\}
\end{aligned}
$$

where ' $\mathrm{s}_{n}:\left\{p_{1} \ldots p_{n}\right\}$ ' is a shorthand for 'situation $\mathrm{s}_{n}$ verifies propositions $p_{1} \ldots p_{n}$.' Nevertheless, there are other situations that are candidates for representing actuality. The first one expresses the fact that the system is in state (2), namely, situation $\mathrm{s}_{4}$ which makes true only $\left(p_{x^{+}}\right)$. Given (ECA), if a situation that verifies only a proposition that describes the state of the system as having spin up (down) along the $z$-axis is a candidate for representing actuality, it follows that a situation that makes true only a description of the system as being in a superposition of spin along the $x$-axis also must be a candidate for representing actuality. Let us capture this fact with the following propositions:

$\left(s p_{x}^{+}\right)$The system is in a superposed state of being spin up plus spin down along the $x$-axis.

$\left(s p_{x}^{-}\right)$The system is in a superposed state of being spin up minus spin down along the $x$-axis.

According to (ECA) then, there must be two situations, $s_{5}$ and $\mathrm{s}_{6}$, that verify, respectively, $\left(s p_{x}^{+}\right)$and $\left(s p_{x}^{-}\right) .{ }^{30}$

Therefore, the list of the possible situations that are candidates for representing actuality is the following:

$$
\begin{aligned}
& \mathrm{s}_{1}:\left\{p_{z^{+}}\right\} \\
& \mathrm{s}_{2}:\left\{p_{z^{-}}\right\} \\
& \mathrm{s}_{3}:\left\{p_{z^{+}}, p_{z^{-}}\right\} \\
& \mathrm{s}_{4}:\left\{p_{x^{+}}\right\} \\
& \mathrm{s}_{5}:\left\{s p_{x}^{+}\right\} \\
& \mathrm{s}_{6}:\left\{s p_{x}^{-}\right\}
\end{aligned}
$$

\footnotetext{
30 One may wonder why this is not enough to show that Darby and Pickup's account fails to model quantum phenomena. If the model considers as candidates for actuality situations that make true propositions like $\left(s p_{x}^{+}\right)$and $\left(s p_{x}^{-}\right)$, then it clearly fails. Since the first assumption has been that the system in the actual world has a determinate state of spin, these propositions — or so the thought goes—determinately misrepresent the actual world. This argument has some mordent, especially because situations are concrete parts of the actual world, rather than ersatz. That being said, an assumption of their argument is that an indeterminate state of affair is not misrepresented by a determinate one. If one charitably assumes that the logic underlying Darby and Pickup's approach is similar to that of Barnes and Williams (2011), then also the converse should hold. That is, an indeterminate state of affairs does not determinately misrepresent a determinate one. I come back on this later on (Sect. 5), when I discuss how one could resist the idea that $\mathrm{s}_{5}$ and $\mathrm{s}_{6}$ are candidates for actuality. I thank an anonymous referee for a discussion on this point.
} 
Now my argument against Darby and Pickup can be easily stated. Recall again $\left(p_{x^{+}}\right)$: the system is in a state that is an eigenvector of the operator $\sigma_{x}$ with a corresponding eigenvalue +1 . Such a proposition seems to be determinately true, insofar as one of the premises of the whole argument is that, in the actual concrete world, our electron is in a state (1). According to the Darby and Pickup model, a proposition is determinately true if it is true in at least one situation (candidate for representing actuality) but false in no other situation. However, when we evaluate $\left(p_{x^{+}}\right)$according to Darby and Pickup's account, it turns out to be metaphysically indeterminate. Indeed, it is true at $\mathrm{s}_{4}$, but falsified by $s_{5}$ and $s_{6}$. When a system is in a superposed state, then necessarily its state is not an eigenstate of the corresponding operator, whose eigenvectors (belonging to different eigenvalues, as in the present context) are the terms of the superposition. Given that it is not metaphysically indeterminate in the actual world if the system is in state spin up along the $x$-axis, Darby and Pickup's approach wrongly accounts this case as metaphysically indeterminate. It follows that Darby and Pickup's account is not reliable for modelling quantum indeterminacy.

\section{Is the argument conclusive?}

The argument proposed hinges on some assumptions that might be resisted. In what follows, I will briefly discuss some ways of doing so. The conclusion of this section is that, in the best-case scenario, blocking my argument commits one to claims that are unlikely to be willingly endorsed by friends of MI.

Excluding the assumptions of Darby and Pickup's view, my argument hinges on three main points; it goes without saying that the most straightforward way of resisting it is that of challenging them. These three additions to Darby and Pickup's account are: (i) the admissibility of $s_{4}$ as a candidate for representing actuality, (ii) the claim that $\left(p_{x^{+}}\right)$is false in $\mathrm{s}_{5}$ and $\mathrm{s}_{6}$, and (iii) the admissibility of $\mathrm{s}_{5}$ and $\mathrm{s}_{6}$ as candidates for representing actuality.

(i) The admissibility of $s_{4}$ :

A first way of blocking the argument above is to deny that the situation in which the system has a definite spin up along the $x$-axis, i.e. $s_{4}$, might be a candidate for representing actuality. The reasoning would be something along the following lines: when there is MI, one has to consider only those situations that deal with MI. Therefore, since the spin along the $x$-axis is determined, the only relevant situations are those that deal with the $z$ spin. Such an argument would be not only highly counter-intuitive but it would betray the whole spirit of situation semantics. Indeed, Darby and Pickup's account is supposed to differentiate metaphysically determined and indeterminate states of affairs. Therefore, if in the actual world it is determinately the case that $p$ obtains, then there must be a situation at which $p$ is true. From this, it follows that, insofar as in our example the system determinately has spin up along the $x$-axis, the proposition $\left(p_{x^{+}}\right)$must be true at least at a situation that is a candidate for representing actuality. Therefore, since $\mathrm{s}_{4}$ verifies only $\left(p_{x^{+}}\right)$and does not misrepresent reality in 
any other regards, it must be a candidate for representing actuality. ${ }^{31}$ If it were not, indeed, the situation semantics proposed by Darby and Pickup would be unable to account for the fact that, in the actual world, it is determinately the case that the electron has spin up along the $x$-axis.

(ii) The truth value of $\left(p_{x^{+}}\right)$in $s_{5}$ and $s_{6}$ :

An alternative way to resist my argument would be to insist that $\left(p_{x^{+}}\right)$is neither true nor false at $\mathrm{s}_{5}$ and $\mathrm{s}_{6}$. Indeed, one might want to argue that, when the system is in a superposed state, it is neither true nor false that it has the property of having spin up (or down) along the relevant axis. Insofar as $\left(p_{x^{+}}\right)$is supposed to capture such a property, then it should be neither true nor false at $s_{5}$ and $s_{6}$. Indeed, one might claim, such a reply is really natural because it makes $\left(p_{x^{+}}\right)$determinately true, ${ }^{32}$ as it should. Now, such a reply hinges on an ambiguity between the mathematical description of the state of a system and the property that the physical system instantiates. I address such an ambiguity in the final part of the next paragraph; the considerations that I make there elucidate why I take $\left(p_{x^{+}}\right)$to be falsified by $\mathrm{s}_{5}$ and $\mathrm{s}_{6}$. Before turning to that, I think it is worth it to point out that, even granting that $\left(p_{x^{+}}\right)$is neither true nor false at $\mathrm{s}_{5}$ and $s_{6}$, my argument still points out two main worries about the Darby and Pickup account.

Firstly, it seems that if $\left(p_{x^{+}}\right)$is neither true nor false at $s_{5}$ and $s_{6}$, then these situations include a metaphysically indeterminate state of affairs, i.e. the electron being in a superposition of being spin up and down. How can we understand such indeterminacy? Following Darby and Pickup's account, these situations should be made each of two other situations, one at which the system has spin up along the $x$ axis and one at which it has spin down. Such an understanding is suspicious for many reasons. On the one hand, the situation at which the system has spin down along the $x$-axis does not seem admissible, insofar as it determinately misrepresents the actual world. On the other, such a move will open an infinite regression of precisifications, which doubtfully helps us understand quantum indeterminacy. ${ }^{33}$

Another difficulty is the following. Even granting that $\left(p_{x^{+}}\right)$is neither true nor false at $s_{5}$ and $s_{6}$, it still remains that, in their account, a determinate state of affairs in the actual world is understood as worldly indecision, so to speak, between different possible indeterminate states of affairs. The upshot of my argument is that they are committed to the view that when a system has determinately spin up along the $x$ axis, the world is unsettled between a superposition of spin up plus spin down along the $x$-axis (the situation $\mathrm{s}_{5}$ ) and a superposition of spin up minus spin down along the $x$-axis (the situation $\mathrm{s}_{6}$ ). When the system is superposed, it might make intuitive sense to claim that the world is unsettled between two definite possibilities. However,

\footnotetext{
31 The fact that $\left(p_{x^{+}}\right)$must be true at some situation is more than enough; indeed even if one successfully denies the admissibility of $\mathrm{s}_{4}$ (for example claiming ad hoc that a situation must verify more than a proposition), there must be nonetheless a situation that makes true $\left(p_{x^{+}}\right)$. In that case, it is trivial to substitute to my $\mathrm{s}_{4}$ that situation, and run the argument with it.

32 Since it would be verified by at least a situation, namely $\mathrm{s}_{4}$, and falsified by no other.

33 Such a consequence has been pointed out for other meta-level accounts as well by Calosi and Wilson (2018, p. 2615). One way of resisting it is denying the possibility of representing higher-order vagueness; but since representing higher-order vagueness is a feature that friends of meta-level approach would not give up, I will not consider such a reply further.
} 
claiming that a determinate state can be understood as worldly indecision between indeterminate states seems plainly odd and obscure. Even if it is not a knockdown argument, I claim that a view with such a consequence is highly unappealing. In order to avoid these worries, rather than accepting that $\left(p_{x^{+}}\right)$is neither true nor false at s5 and $s_{6}$ it is more likely that friends of Darby and Pickup's account would be tempted to deny $s_{5}$ and $s_{6}$ as admissible candidates for representing actuality. We finally turn to this kind of reply.

(iii) The admissibility of $s_{5}$ and $s_{6}$ :

There are three main ways of challenging the admissibility of $s_{5}$ and $s_{6}$ as candidates for representing actuality.

The first way consists of claiming that $s_{5}$ and $s_{6}$ are not possible because they determinately misrepresent the actual world: since the system is in a determinate state of spin along the $x$-axis, being in a superposed state determinately misrepresents the actual world. Note that if this were the case, then the converse should also hold: if a system is superposed in reality, then a determinate state misrepresents it. Consequently, Darby and Pickup's account would instantly fail, insofar as accounting superposition as worldly indecision between spin up and spin down is the core of their explanation. In other words, if a superposed state along the $x$-axis misrepresents the actual world, given its mathematical equivalence with being spin up (down) along the $z$-axis, then there would no longer be any reason to claim that $s_{2}$ and $s_{3}$ are candidates for representing actuality. Such a reply would make my argument invalid, but at the prize of rejecting the Darby and Pickup account. Moreover, this move would be in opposition to the very way in which meta-level approaches see quantum indeterminacy. Indeed, according to these accounts of MI, when a system is superposed between spin up and down along an axis, the spin of the system is metaphysically indeterminate. Such indeterminacy is understood as it is not determinately the case that the system has spin up and it is not determinately the case that the system has not spin up (Darby 2010). ${ }^{34}$ If this is the way in which defenders of meta-level accounts understand quantum indeterminacy, claiming that the proposition $\left(p_{\alpha}\right)$ 'it is determinately the case that the system is in state spin up along the $\alpha$ axis' misrepresents a superposed state begs the question against their approach to MI. According to them indeed, when a system is in a superposed state, its logical description is ' $\neg \Delta p_{\alpha} \wedge \neg \Delta \neg p_{\alpha}$, where ' $\alpha$ ' is any axis and ' $\Delta$ ' is the determinacy operator that must be read as 'it is determinate that'. ${ }^{35}$ A proposition that captures such a logical structure is not determinately misrepresented, nor determinately misrepresents, states of affairs captured by ' $\Delta p_{\alpha}$ ' and ' $\Delta \neg p_{\alpha}$ '.

A second way to deny the admissibility of $s_{5}$ and $s_{6}$ as candidates for representing actuality is that of directly rejecting the validity of (ECA). I think that such a move would make Darby and Pickup's account highly unappealing; indeed, what can possibly be the justification for rejecting (ECA)? I see why, in epistemic evaluations, for instance, the sobstitutio salva veritate might fail. For example, if Bob does not

\footnotetext{
34 Note that a big assumption of their approach is that having spin down is equal to the negation of 'having spin up'. I think that such an assumption could be challenged, especially for observables with continuous values (such as position and momentum), albeit I will not do that here.

35 See Fine (1975) for one way of introducing such a modal operator and Darby (2010) for the application to quantum cases.
} 
know that Alice's dog is named 'Nala', then the proposition 'Bob knows that Alice is playing with her dog' might be true at the same time that 'Bob knows that Alice is playing with Nala' being false. Nevertheless, in the case at hand, we are dealing with mathematically equivalent descriptions of the very same state, and we are concerned with the metaphysical descriptions. In other words, we are dealing with a de re reading of the propositions: we are talking about the referents of the terms involved, rather than the terms themselves. And in a de re reading, 'Bob knows that Alice is playing with her dog' and 'Bob knows that Alice is playing with Nala' are also equally true. Therefore, I do not see how one can argue that a situation that verifies 'the system is in state spin up along the $z$-axis' might be a candidate for representing actuality, and one that makes true 'the system is in a superposition of spin up and down along the $x$-axis' could not: spin up along the $z$-axis describes the same state of 'being in a superposition along the $x$-axis'. Moreover, denying the equivalence leaves space for rejecting the very first assumption of my argument, i.e. that a system with a definite spin along the $x$-axis is in a superposed state along the $z$-axis. Since such a fact is a well-established experimental result, I take every view that allows its rejection as at least suspicious.

I turn now to the last way of resisting my argument, which shows that I may have left room for ambiguities in the formulation of my argument. Such a consideration helps to develop a stronger version of the argument, in that I think it highlights what is - generally speaking — suspicious about using meta-level accounts of MI to explain quantum indeterminacy. One might want to point out that the situations used by Darby and Pickup are incomplete but definite, that is to say, they do not contain in themselves any indeterminacy of the sort. Such a claim might be used, once again, for undermining my claim that $\mathrm{s}_{5}$ and $\mathrm{s}_{6}$ are admissible situations: insofar as these propositions describe indeterminate states of affairs, they cannot be verified by a situation that is a candidate for representing actuality. Moreover, one might object that, insofar as Darby and Pickup explicitly claim that their situations are incomplete but definite, I have misunderstood their account all along. Such a reply, however, hinges on the ambiguity mentioned earlier, to which we now turn. The ambiguity lies between propositions that refer to the mathematical description of the quantum state and propositions that describe the properties instantiated by the quantum objects. Consider the following propositions:

$\left(p_{x^{+}}\right)$The system is in a state that is an eigenvector of the operator $\sigma_{x}$ with a corresponding eigenvalue +1 .

(su) The electron has the property of having spin up along the $x$-axis.

Propositions $\left(p_{x^{+}}\right)$and $(s u)$ should be, given the eigenvalue-eigenstate link, equivalent; indeed, (EEL) implies that they should always have the same truth value. The very core of (EEL) is exactly that of connecting the mathematical description of a quantum system to its metaphysical one, i.e. which properties it instantiates. Nonetheless, according to meta-level approaches, these descriptions are not equivalent. Reasonably indeed, when the latter is metaphysically indeterminate (for example when the system is in a superposition of spin along the $x$-axis), the former is determinately false: when a system is in a superposed state of spin, even conceding that the proposition that captures the fact that the system has spin up (or spin down) is indeterminate, the proposition that says that the state of the system is mathematically describable as an 
eigenvector of the corresponding operator is determinately false. In fact, propositions that refer to the mathematical description of the state of the system are never indeterminate: when the electron is in a state of superposition of its spin along the $x$-axis, the proposition 'the electron is in state $|\psi\rangle=\frac{1}{\sqrt{2}}\left(|\uparrow\rangle_{x} \pm|\downarrow\rangle_{x}\right)$ ' is always determinately true. What is indeterminate is whether, when the system is in such a state, it instantiates the property of having spin up (down). I think that meta-level approaches are committed to accepting that propositions that describe the mathematical state of the system might be indeterminate; as I have done throughout the whole paper, I stick only to Darby and Pickup's account in what follows. ${ }^{36}$

So, I would concede that accepting that $\mathrm{s}_{5}$ and $\mathrm{s}_{6}$ as admissible might be suspected of begging the question against Darby and Pickup's view: the core of their account is exactly that such an indeterminate instantiation of properties must be captured by a disagreement of possible situations; and therefore, a situation cannot contain indeterminate states of affairs. Nevertheless, this is of no help for the friends of their account because they must concede that the following proposition:

$\left(i_{z}\right)$ The electron is in a state that is not an eigenvector of the operator $\sigma_{z}$; rather, the system is in a state that might be expressed as a linear combination of the eigenvectors of $\sigma_{z}$ with respective eigenvalues of +1 and -1 ,

which is determinately true or false, might be verified by a situation that is a candidate for actuality. If this is so, the argument becomes even stronger than before: in the actual world the system is in a definite state of spin along the $x$-axis, and so it is in a superposition of spin along the $z$-axis. That is to say, $\left(i_{z}\right)$ should be determinately true. Recall that in this case, Darby and Pickup say that there are two possible situations that are candidates for representing actuality, one that makes true that the system has spin up along $z\left(\mathrm{~s}_{7}\right)$, and another that verifies that the system has spin down $\left(\mathrm{s}_{8}\right) .{ }^{37}$ If $s_{7}$ and $s_{8}$ are candidates for representing actuality, then, given (EEL) and (ECA), I should be free to admit as candidates for representing actuality those situations that make true the mathematical descriptions of having spin up and down along the $z$-axis, that is to say, $s_{1}$ and $s_{2}$. However, this implies a contradiction again. In the actual world the system is in a superposition of spin along the $z$-axis; therefore, $\left(i_{z}\right)$ should be determinately true. Given the truth of $\left(i_{z}\right)$, it should follow that both $\left(p_{z^{+}}\right)$and $\left(p_{z^{-}}\right)$ are determinately false. ${ }^{38}$ These propositions should be determinately false because in the actual world the system is not in a state that is an eigenstate of the operator $\sigma_{z}$. Therefore, they should not be true at any possible situation (but false in at least one). According to the Darby and Pickup account though, they turn out to be indeterminate, insofar as $\left(p_{z^{+}}\right)$is made true by $s_{1}$ and falsified by $s_{2}$, where $\left(p_{z^{-}}\right)$is false at the

\footnotetext{
${ }^{36}$ A way out might be that of giving up the (EEL) for a different version of the link. The viability of such a move and the possibility of generalizing such a claim to all meta-level approaches are the subject of a current project.

37 Situation $\mathrm{s}_{7}$ makes true only the proposition 'the system has spin up along the $z$-axis', and $\mathrm{s}_{8}$ verifies only the proposition 'the system has spin up along the $z$-axis'. To be as charitable as possible, I assumed they are different from $\mathrm{s}_{1}$ and $\mathrm{s}_{2}$, insofar as $\left(p_{z^{+}}\right)$and $\left(p_{z^{-}}\right)$are different propositions from those verified by $\mathrm{s}_{7}$ and $\mathrm{s}_{8}$. Given (EEL), these propositions are equivalent.

38 Provided some semantic assumption according to which, $\neg p$ being true entails $p$ being false, and vice versa. Insofar as Darby and Pickup (2019, p. 11) endorse these assumptions, I did assume them unproblematically.
} 
former situation and true at the latter. By the same token, $\left(i_{z}\right)$ also receives a wrong truth-value. Whereas in the actual world it should be determinately true, it turns out to be false, insofar as it is false at both $s_{1}$ and $s_{2}$, and it is not verified by any situation that is a candidate for representing actuality. ${ }^{39}$

The only way, that I see at least, for resisting this last conclusion is to accept that propositions like $\left(p_{z^{+}}\right),\left(p_{z^{-}}\right)$and $\left(i_{z}\right)$ might have an indeterminate truth value. But this amounts to accepting that the mathematical description of the quantum state (i.e. the mathematical description of the properties instantiated by quantum objects) is unreliable for drawing metaphysical conclusions. Indeed, such a claim would amount to either (a) the claim that, when a system is in a superposed state, then it is indeterminate which eigenvector represents the state (and so $\left(p_{z^{+}}\right)$and $\left(p_{z^{-}}\right)$have an indeterminate truth value), ${ }^{40}$ or (b) a rejection of the eigenvalue-eigenstate link. Alas, these claims are worrisome for the friends of MI. (a) is dangerously close to an epistemic understanding of the formalism of quantum mechanics. As a matter of fact, the claim that mathematical descriptions do not tell us the exact properties of quantum systems has been considered the hallmark of epistemic interpretations of the quantum states. I would be happy to concede that such a view is the right one if forced by conclusions drawn by the formalism of quantum mechanics, established experiments or mathematical theorems. Nonetheless, if a view of MI would force me to accept such a position on the quantum states, I would rather move to another account of MI. And the reason for that is soon explained: examples of MI arising in quantum mechanics are drawn precisely by taking the formalism at face value. A metaphysical account that accepts that the quantum states do not describe the properties of a quantum system correctly undermines, from the beginning, the very reason for looking for quantum MI. ${ }^{41}$ The same happens with (b) since, as we have seen, (EEL) is the principle that justifies the claim that there is quantum MI in the first place. An account that accepts either (a) or (b) (or both), rejects beforehand the very idea that quantum mechanics presents metaphysically indeterminate states of affairs. As such, it is not something that an account of MI should embrace.

\footnotetext{
39 Even if they would find a situation at which it is true, according to their account it would still turn out to be indeterminate, rather than determinately true as it should; indeed, the fact that $\left(i_{z}\right)$ is false at $\mathrm{s}_{1}$ and $s_{2}$ excludes the possibility of it being determinately true.

40 In other words, that we use superposed states to represent the fact that the system always is either in one eigenvector or in the other. The only way to make sense of this claim is to read it epistemically: we use superposition to represent the fact that an electron, say, has either spin up or spin down at all the times, but we ignore in which state the electron is until we measure it. Friends of MI are, as we have seen in Sect. 2, committed to some form of ontic understanding of the quantum state. Therefore, showing that this way of resisting my argument commits one to an epistemic reading of the quantum state is more than enough to rule it out (as a possible counterargument available to the friends of Darby and Pickup's account).

41 A possible way out would be to endorse a radical view according to which the truth-values of mathematical descriptions are ruled by quantum logic. Since it is commonly assumed that the meta-language of the mathematical formalism of a physical theory is classical logic (or intuitionistic, at best), I do not consider such a possibility further. However, it would be interesting to see whether there are arguments in favour of the adoption of such a meta-logical framework for the quantum formalism that are independent of one's account of MI. I thank an anonymous referee for bringing up this interesting point.
} 


\section{Conclusion}

Under some controversial assumptions, it is possible to argue that non-relativistic quantum mechanics offers a genuine example of metaphysical indeterminacy. As we have seen, given that there are two main approaches to MI in the literature, many philosophers try to test their compatibility with quantum cases. Since the fall of the champion of meta-level approaches proposed by Barnes and Williams, many have tried to develop alternative supervaluationist theories able to cash out quantum indeterminacy.

In the paper, I have argued that the last of these proposals, namely Darby and Pickup (Darby and Pickup 2019)'s view, fails in the context of quantum mechanics. In this theory, the mathematical description of a state of definite spin along an axis is mathematically equivalent to the description of being in a superposition on another axis. I showed that, when one considers some propositions concerning the mathematical description of a quantum system, Darby and Pickup's account assigns them a wrong truth-value. Moreover, I argued that the most straightforward ways of resisting my argument imply a rejection of one of the assumption needed to claim that there is quantum indeterminacy in the first place. As with Barnes and Williams' view, showing that there is at least one quantum case in which their account fails is more than enough to inspire doubt concerning its tenability in the quantum arena.

I have taken Darby and Pickup's account as an example. But such an example is interesting because it hints at the fact that there might be a reason why their account, as well as that proposed by Barnes and Williams previously, fails. And such a reason I think lies at the heart of meta-level approaches and the irremediable incompatibility of their core tenet with quantum mechanics. The interesting question then would be whether it is possible to generalize my argument to any other meta-level account of MI. If such a generalization is possible, this would mean that object-level accounts should be the preferred tool for understanding MI (which in turns would imply the question 'Which object-level account, if any, deals with quantum indeterminacy?'). My intuition is that such a generalization of my argument is possible. ${ }^{42}$ The (EEL) tightly links the mathematical and the metaphysical description. Since meta-level approaches treat MI as an indeterminacy of the truth value of the propositions describing it, and (EEL) bridges these propositions in natural language to the mathematical ones, friends of meta-level accounts seem to be committed to the idea that also the mathematical description of quantum states might be indeterminate. Accepting that the mathematical description of quantum systems is not precise (i.e. we cannot take it at face value) seems dangerously close to forms of $\psi$-epistemic understanding of quantum formalism. As a matter of fact, a $\psi$-epistemic reading of the quantum state is clearly incompatible with quantum MI. Indeed, the very first reason for looking at quantum MI was that of taking quantum formalism at face value.

I think that trying to generalize the argument here proposed is essential. Given the popularity of meta-level approaches, one way of stopping the industry of introducing ad hoc modifications to Barnes and Williams' account is that of showing once and for

\footnotetext{
$\overline{42}$ Even if it is far from trivial. Especially for accounts, like the one put forward by Torza (2017), that does not treat MI as worldly indecision.
} 
all that as long as one is willing to take quantum states as real, he/she must admit that quantum indeterminacy is not worldly indecision.-

Acknowledgements For helpful comments and discussions on earlier drafts of the paper, I would like to thank: Stefano Bonzio, Enrico Cinti, Michael Esfeld, Vincenzo Fano, Pierluigi Graziani, Niels Linnemann, Andrea Oldofredi, Cristian Mariani, Robert Michels, Marco Sanchioni, Frida Trotter, Christian Wüthrich, and three anonymous referees. Last but not least, I am thankful to Claudio Calosi, whom I stressed with infinite discussions about the paper.

Funding Open Access funding provided by Université de Genéve

\section{Compliance with ethical standards}

Conflict of interest The authors declare that they have no conflict of interest.

Open Access This article is licensed under a Creative Commons Attribution 4.0 International License, which permits use, sharing, adaptation, distribution and reproduction in any medium or format, as long as you give appropriate credit to the original author(s) and the source, provide a link to the Creative Commons licence, and indicate if changes were made. The images or other third party material in this article are included in the article's Creative Commons licence, unless indicated otherwise in a credit line to the material. If material is not included in the article's Creative Commons licence and your intended use is not permitted by statutory regulation or exceeds the permitted use, you will need to obtain permission directly from the copyright holder. To view a copy of this licence, visit http://creativecommons.org/licenses/by/4.0/.

\section{References}

Akiba, K. (2004). Vagueness in the world. Nô̂s, 38(3), 407-429.

Akiba, K. (2015). How Barnes and Williams have failed to present an intelligible ontic theory of vagueness. Analysis, 75(4), 565-573. https://doi.org/10.1093/analys/anv074.

Albert, D. Z., \& Loewer, B. (1996). Tails of schrödinger's cat. In: Clifton, R. (ed.), Perspectives on quantum reality: Non-relativistic, relativistic, and field-theoretic (pp. 81-92). Springer, Netherlands. https:// doi.org/10.1007/978-94-015-8656-6_7.

Barnes, E., \& Cameron, R. (2009). The open future: Bivalence, determinism and ontology. Philosophical Studies, 146(2), 291-309. https://doi.org/10.1007/s11098-008-9257-6.

Barnes, E., \& Williams, J. R. G. (2011). A theory of metaphysical indeterminacy. In K. Bennett \& D. W. Zimmerman (Eds.), Oxford Studies in Metaphysics (Vol. 6, pp. 103-148). Oxford University Press, Oxford.

Bokulich, A. (2014). Metaphysical indeterminacy, properties, and quantum theory. Research Philosophica, 91(3), 449-475. https://doi.org/10.11612/resphil.2014.91.3.11.

Calosi, C., \& Mariani, C. (2020). Quantum relational indeterminacy. Studies in History and Philosophy of Science Part B: Studies in History and Philosophy of Modern Physics, 71, 158-169. https://doi.org/ 10.1016/j.shpsb.2020.06.002.

Calosi, C., \& Wilson, J. (2018). Quantum metaphysical indeterminacy. Philosophical Studies, pp. 1-29.

Darby, G. (2010). Quantum mechanics and metaphysical indeterminacy. Australasian Journal of Philosophy, 88(2), 227-245. https://doi.org/10.1080/00048400903097786.

Darby, G., \& Pickup, M. (2019). Modelling deep indeterminacy. Synthese, pp. 1-26.

Darby, G., Pickup, M., \& Robson, J. (2017). Deep indeterminacy in physics and fiction. In O. Bueno, S. French, G. Darby, \& D. Rickles (Eds.), Thinking about science, reflecting on art, bringing aesthetics and philosophy of science together. London: Routledge.

Daumer, M., Dürr, D., Goldstein, S., \& Zanghì, N. (1996). Naive realism about operators. Erkenntnis, 45(2-3), 379-397.

Evans, G. (1978). Can there be vague objects? Analysis, 38(4), 208. https://doi.org/10.1093/analys/38.4. 208. 
Fine, K. (1975). Vagueness, truth and logic. Synthese, 30(3-4), 265-300. https://doi.org/10.1007/ BF00485047.

French, S., \& Krause, D. (2006). Identity in physics: a historical, philosophical, and formal analysis. Oxford: Oxford University Press.

Gleason, A. M. (1957). Measures on the closed subspaces of a hilbert space. Journal of Mathematics and Mechanics, 6(6), 885-893.

Glick, D. (2017). Against quantum indeterminacy. Thought: A Journal of Philosophy 6(3), 204-213.

Kochen, S., \& Specker, E. P. (1967). The problem of hidden variables in quantum mechanics. Journal of Mathematics and Mechanics, 17(1), 59-87.

Levesque, H. J. (1984). A logic of implicit and explicit belief. In Proceedings of the fourth AAAI conference on artificial intelligence. AAAI Press, AAAI'84 (pp. 198-202).

Lewis, D. K. (1986). On the plurality of worlds. Wiley-Blackwell, London

Lewis, P. J. (2016). Quantum ontology: A guide to the metaphysics of quantum mechanics. Oxford University Press, USA.

Lowe, E. J. (1994). Vague identity and quantum indeterminacy. Analysis, 54(2), 110-114. https://doi.org/ 10.1093/analys/54.2.110.

Mariani, C., Michels, R., \& Torrengo, G. Against quantum mechanics based objections to metaphysical supervaluationism. Manuscript.

McMullin, E. (1984). A case for scientific realism. In: Leplin, J. (Ed.), Scientific realism. University of California, California, pp. 8-40.

Pusey, M. F., Barrett, J., \& Rudolph, T. (2012). On the reality of the quantum state. Nature Physics, $8(6)$, 475-478. https://doi.org/10.1038/nphys2309.

Reichenbach, H. (1975). Three-valued logic and the interpretation of quantum mechanics. In Hooker, C. A. (Ed.), The logico-algebraic approach to quantum mechanics: Volume I: Historical evolution (pp. 53-97). Springer, Dordrecht. https://doi.org/10.1007/978-94-010-1795-4_4.

Russell, B. (1923). Vagueness. Australasian Journal of Philosophy, 1(2), 84-92. https://doi.org/10.1080/ 00048402308540623 .

Skow, B. (2010). Deep metaphysical indeterminacy. Philosophical Quarterly, 60(241), 851-858.

Smith, N. J. J., \& Rosen, G. (2004). Worldly indeterminacy: A rough guide. Australasian Journal of Philosophy, 82(1), 185-198. https://doi.org/10.1080/713659795.

Torza, A. (2017). Quantum metaphysical indeterminacy and worldly incompleteness. Synthese. https://doi. org/10.1007/s11229-017-1581-y.

Tye, M. (1990). Vague objects. Mind, 99(n/a), 535. https://doi.org/10.1093/mind/XCIX.396.535.

Wallace, D. (2019). What is orthodox quantum mechanics? In Cordero, A. (Ed.), Philosophers look at quantum mechanics. Springer, Berlin.

Williamson, T. (1994). Vagueness. London: Routledge.

Wilson, A. (2020). The nature of contingency: Quantum physics as modal realism. Oxford: Oxford University Press.

Wilson, J. M. (2013). A determinable-based account of metaphysical indeterminacy. Inquiry: An Interdisciplinary Journal of Philosophy 56(4), 359-385. https://doi.org/10.1080/0020174x.2013.816251.

Wolff, J. (2015). Spin as a determinable. Topoi, 34(2), 379-386. https://doi.org/10.1007/s11245-015-93192 .

Publisher's Note Springer Nature remains neutral with regard to jurisdictional claims in published maps and institutional affiliations. 